\title{
Dose-Efficient Cryo-STEM Imaging of Vitrified Biological Samples
}

\author{
Katherine Spoth ${ }^{1}$, Yue Yu ${ }^{1}$, Kayla Nguyen ${ }^{2}$, Zhen Chen $^{1}$, David Muller ${ }^{1}$ and Lena Kourkoutis ${ }^{1}$ \\ ${ }^{1}$ Cornell University, Ithaca, New York, United States, ${ }^{2}$ University of Illinois Urbana-Champaign, Urbana, \\ Illinois, United States
}

Cryo-transmission electron microscopy (cryo-TEM) has enabled new insights into biology as it allows the study of specimens preserved in their near-native state through vitrification. Cryo-scanning TEM (cryoSTEM) on the other hand, has rarely been used in the life science. Early attempts at cryogenic imaging showed fewer details in STEM mode than were accessible by TEM [1]. A reason for this deficiency is that traditional STEM imaging schemes do not make efficient use of the incident beam. Annular dark field imaging discards the entire forward-scattered beam, while coherent bright field (BF) imaging utilizes only a small detector located on the optical axis to form a phase-contrast image (Fig. 1a). Even with these signals collected in parallel a significant number of the incident electrons are discarded. This makes STEM imaging difficult for radiation sensitive specimens, such as frozen-hydrated material. Despite that, previous studies have demonstrated the potential for cryo-STEM imaging with carefully optimized detector geometries, allowing tomography of whole cells [2].

The development of new pixelated direct detectors optimized for STEM creates new possibilities for STEM imaging with higher dose-efficiency. We make use of the electron microscope pixel array detector (EMPAD) to collect a convergent beam electron diffraction (CBED) pattern at every scan position, detecting nearly all electrons incident on the specimen, and resolved by both position and scattering angle [3]. The resulting 4D dataset allows flexible choice of scattering angles for image generation as well as new types of imaging modes not possible with a traditional setup.

Here, we will discuss tilt-corrected bright-field STEM (tcBF-STEM), a dose efficient imaging technique which allows us to form a coherent STEM image using all the electrons in the bright-field disk [4,5]. The EMPAD acts as an array of coherent detectors - each pixel is small compared to the convergence angle of the beam and those located within the BF disk each produce a phase-contrast image. By carefully combining the images produced by each pixel in the BF disk, taking into account the effect of defocus aberrations, we obtain a coherent image that utilizes significantly more of the incident beam than a conventional BF image (Fig. 1). We demonstrate tcBF-STEM on thin and thick frozen-hydrated biological specimen. For thick samples, we compare images of E. coli acquired using the tilt-corrected bright field mode with TEM and zero-loss EFTEM imaging and demonstrate that tcBF-STEM outperforms these methods at electron doses appropriate for projection imaging and for a cryo-electron tomography (Fig. 1). tcBF-STEM is less sensitive to chromatic blur than TEM and more dose-efficient than EFTEM in thick samples. It can also be used in samples too thick for reliable ptychographic reconstructions, i.e., where the strong phase approximation needed for ptychography no longer holds as significant probe propagation has occurred.

In thinner vitreous specimens such as large molecular weight ribosomes and the 10-times-lighter apoferritin complexes a larger fraction of electrons is scattered into the BF region (Fig. 2). The additional signal allows high signal-to-noise imaging with smaller incident dose, important for preserving highresolution structural information. [6] 


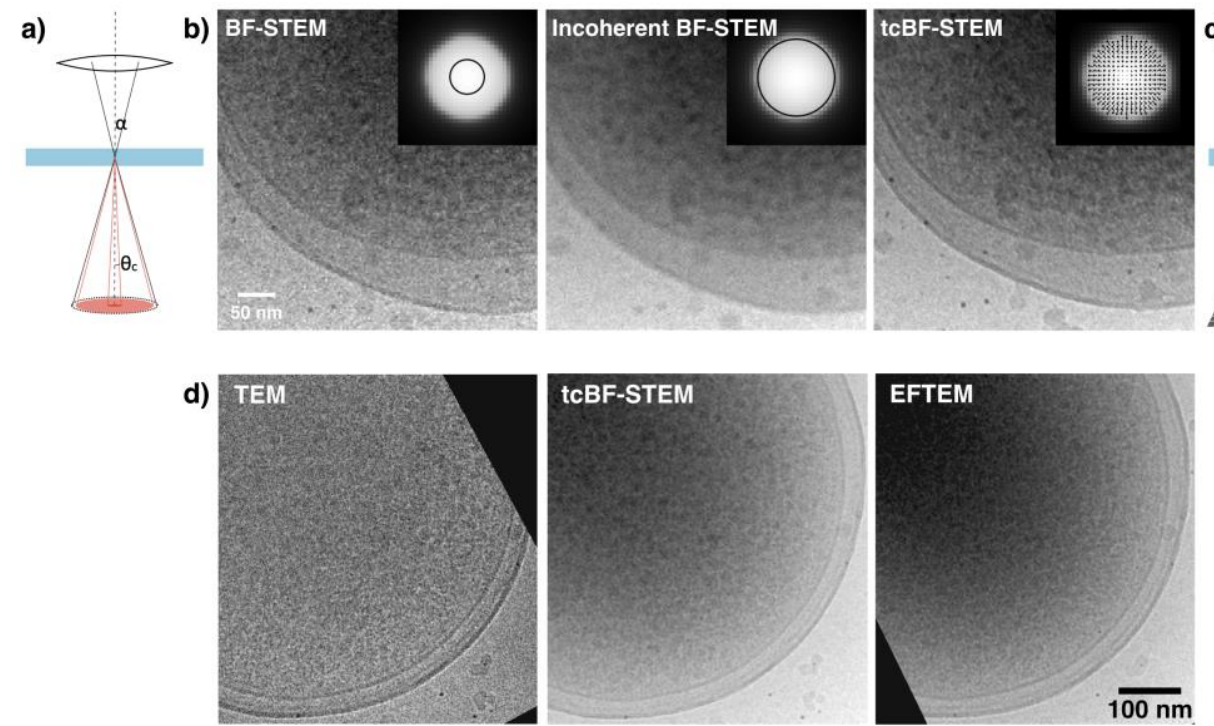

Figure 1. Cryogenic tilt-corrected bright-field STEM (tcBF-STEM) using the EMPAD of frozen-hydrated whole E. coli cells. (a) Coherent STEM imaging with a monolithic detector requires detector angles $(\theta \mathrm{c})$ that are small relative to the size of the BF disk, i.e., the convergence angle $(\alpha)$. (b) A pixelated STEM detector allows conventional detector geometries such as BF-STEM as well as more complex imaging modes including tcBF-STEM. (c) For such a detector each detector pixel within the bright field disk is a coherent STEM detector though located off the optical axis. Off-axis BF-STEM is equivalent by reciprocity to tilted-beam TEM leading to shifts in images formed by individual off-axis BF-STEM detectors. In tcBF-STEM these shifts are corrected for producing high SNR images of even the thickest part of the frozen-hydrated cell. The applied shift map is shown in the inset of (b, right). (d) Comparison of tcBF-STEM imaging using the EMPAD with conventional TEM and zero-loss EFTEM at a dose of 7.5 e/Å2 shows its potential for low-dose tomographic imaging.
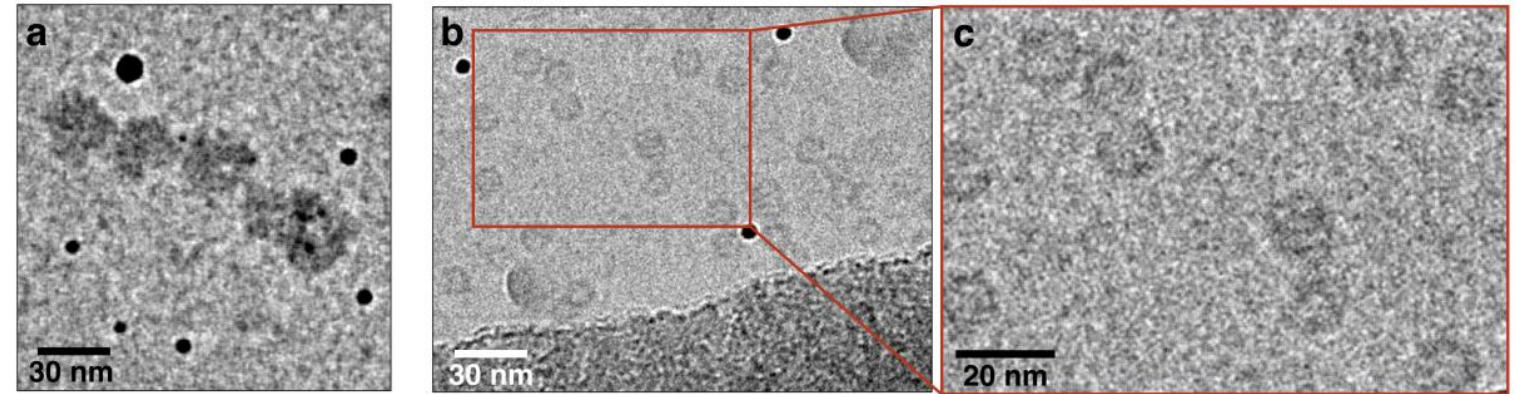

Figure 2. Cryogenic tcBF-STEM imaging of vitrified macromolecules with the EMPAD. (a) Ribosomes imaged at a dose of $\sim 30 \mathrm{e}-/ \AA \mathrm{A} 2$. Dark regions are gold fiducials used for focusing. (b-c) Cryogenic tcBFSTEM also resolves apoferritin complexes which are $\sim 1 / 10$ the molecular weight of ribosomes.

\section{References}

[1] R. D. Leapman, S. B. Andrews. Journal of Microscopy 161 (1991), p. 3-19.

[2] S. G. Wolf, et al., Nat. Meth. 11 (2014), p. 423-428.

[3] M. W. Tate, et al., Microscopy and Microanalysis 22 (2016), p. 237-249.

[4] K. A. Spoth, et al, Microscopy and Microanalysis 23 (2017), p. 804-805.

[5] K. A. Spoth, et al, Microscopy and Microanalysis 24 (2018), p. 876-877.

[6] This work is supported through by the Packard foundation and NSF (DMR-1539918, DMR-1429155, DMR-1719875). 